\title{
Abordaje de la salud bucal en Cuba
}

\section{Nota Científica}

\author{
Estela de los Ángeles Gispert Abreu 1,a \\ 1 Escuela Nacional de Salud Pública, La Habana, Cuba. \\ a Doctora en Ciencias Estomatológicas.
}

\section{Correspondencia:}

Estela de los Ángeles Gispert Abreu: gisperthoy00@gmail.com Calle 2 no.558 entre 15 y 17 Vedado. Municipio Plaza de la Revolución.

Consejo Nacional de Sociedades Científicas de la Salud,

La Habana - Cuba.

ORCID: 0000-0002-3427-4099

\section{Editora invitada:}

Rosa Ana Melgar Hermoza

Universidad Peruana Cayetano Heredia, Perú.

\section{Oral health approach in Cuba}

Conflicto de intereses: los autores declaran no tener conflictos de interés.

Fuente de financiamiento: autofinanciado.

Recibido: $16 / 03 / 20$

Aceptado: $23 / 03 / 20$

Publicado: 09/05/20

\section{Resumen}

La salud bucal requiere prioridad. Reducir la alta carga de morbilidad de las afecciones representa un gran desafío; en ese camino es válido que se compartan lecciones. El presente artículo dirigido a exponer experiencias del abordaje de la salud bucal en Cuba como bien social y derecho humano, caracterizó la organización e institucionalización de la atención, constituida en práctica organizada dentro del sistema nacional de salud único, con cobertura universal y financiamiento estatal; hizo referencia a programas y acciones nacionales, a la formación de recursos humanos siendo la Estomatología General Integral disciplina integradora apoyada en el análisis de la situación de salud, a su vez especialidad que favorece el actuar integrado dentro de la atención primaria de salud. Los resultados muestran avances. Se concluye que varios factores facilitan abordar la salud bucal como bien social y derecho humano en Cuba, no obstante existen retos para el perfeccionamiento.

Palabras clave: Salud bucal; Salud pública; Atención primaria de salud; Servicios de salud dental; Intersectorialidad (fuente: DeCS BIREME).

\section{Abstract}

Oral health requires priority; a great challenge is to reduce the high burden of disease morbidity, in that way it is valid to share lessons. The aim of this article is to expose experiences of the oral health approach in Cuba as a social good and human right. The article characterized the organization and institutionalization of an organized care practice within the single national health system with universal coverage and state funding. This paper referenced to national programs and actions, human resources training being the Integral General Stomatology an integrative discipline based on the health analysis. Also, the specialty favors the integrated action within the primary health care. The results showed advances. It is concluded that several factors may facilitate addressing oral health as a social good and human right in Cuba; however, its improvement has challenges.

Keywords: Oral health; Public health; Primary health care; Dental health services; Intersectoral collaboration (source: MeSH NLM).

(c) Los autores. Este artículo es publicado por la revista Odontología Sanmarquina de la Facultad de Odontología, Universidad Nacional Mayor de San Marcos. Este es un artículo de acceso abierto, distribuido bajo los términos de la licencia Creative Commons Atribucion - No Comercia_Compartir Igual 4.0 Internacional. (http://creativecommons.org/licenses/by-nc-sa/4.0/) que permite el uso no comercial, distribución y reproducción en cualquier medio, siempre que la obra original sea debidamente citada. 


\section{Introducción}

La salud es reconocida como derecho humano fundamental vinculado a los derechos de alimentación, vivienda, trabajo, educación, no discriminación, acceso a la información y participación desde finales de la década del 40' '. Los servicios de salud deben ofrecer calidad y protección contra daños financieros ${ }^{2}$, por lo que constituye un deber de los estados crear las condiciones que encaucen a materializarlo plenamente de forma igualitaria, a través de normativas, legislaciones y reglamentaciones que impidan prácticas discriminatorias, excluyentes y favorezcan políticas nacionales, capacitación profesional, práctica ética y cooperación internacional ${ }^{3}$.

No obstante, cuando se hacía referencia al derecho a la salud el componente bucal quedaba marginado y no es hasta la 7. ${ }^{\text {a }}$ Conferencia Mundial de Promoción de la Salud celebrada en Nairobi, Kenia en 2009, que se hace un llamado a considerar la salud bucal derecho humano esencial para la salud general y la calidad de vida, por consiguiente se reconoció la necesidad de estimular la promoción de la salud bucal, la prevención de las enfermedades bucales, asimismo el desarrollo de capacidades nacionales y comunitarias hacia la acción integrada en el marco de la atención primaria de salud, con políticas, recursos humanos y financieros apropiados ${ }^{3-7}$.

A pesar de ello en la atención odontológica a nivel global persisten alta carga de morbilidad, discapacidad, falta de acceso, tratamientos costosos, desigualdades entre países y a su interior, crecimiento inquietante de las especializaciones, investigación muy dependiente del financiación de la industria y capacitación inadecuada en salud bucal poblacional, ya que la educación dental se ha desarrollado separada de la educación médica y sanitaria, centrada en la odontología restaurativa y clínica ${ }^{8-10}$, como consecuencia en la práctica profesional prima la actuación acorde a las demandas del mercado; requiriéndose reforzar la responsabilidad ética basada en valores ${ }^{11} \mathrm{y}$ acciones integradas e intersectoriales dentro de la cobertura universal de salud ${ }^{10,12}$. Lo referido motiva a exponer experiencias del abordaje de la salud bucal en Cuba como un bien social y derecho humano.

\section{Caracterización de la organización e institucionalización de la atención de la salud bucal}

En el período previo a 1959 primaba la falta de atención estomatológica a la población pues la profesión, llamada entonces Cirugía Dental, se basaba en la práctica privada y el elitismo profesional, consecuencia de las relaciones socio-económicas imperantes. La mayoría de los cirujanos dentistas ejercían en la provincia de La Habana, fundamentalmente en el área metropolitana y privada, dejando las áreas rurales - que tenían necesidades de salud mayores-, a personal empírico. El sistema de salud carecía de coordinación nacional, las instituciones privadas, caritativas y públicas existían independientes unas de otras, la orientación de los servicios era curativa y la calidad difería, siendo la actividad gratuita limitada, de baja calidad y con escasos recursos humanos, por todo lo cual la cobertura era incompleta.

A partir de 1959 se producen cambios que condujeron a políticas públicas en diferentes esferas, en educación la campaña nacional de alfabetización, en salud la gratuidad de los servicios a nivel nacional y en seguridad social la protección a personas sin recursos ni posibilidades de trabajar; también se implementaron políticas sociales destinadas a la mejora de las condiciones y estilos de vida, entre ellas ofertas de empleo, distribución de alimentos básicos, mejora en las condiciones de las viviendas, entre otras, encauzadas a eliminar la pobreza extrema y asegurar justicia social. Estas políticas recibieron la impronta de la participaron comunitaria e intersectorial y contribuyeron a eliminar barreras tanto objetivas como subjetivas a la salud (incluyendo la bucal), que comenzó a ser valorada de manera integral e integrada como bien social, humano, derecho del pueblo y deber del estado.

Durante la década de 1960 se conformó el Sistema Nacional Estatal de Salud, se establece la obligación de todos los egresados a prestar servicios asistenciales por dos años en las áreas rurales, lo que propició que la atención médica y estomatológica se extendiera a los lugares más intrincados de la geografía insular en una infraestructura sanitaria conformada por unidades sanitarias de atención primaria, policlínicos integrales, hospitales, clínicas estomatológicas, centros de higiene y epidemiología e institutos de investigación. En 1961 se constituyó el Ministerio de Salud Pública (MINSAP), que tiene la rectoría del Sistema Nacional de Salud, en su estructura se halla la Dirección Nacional de Estomatología (actual departamento nacional), unidad organizativa en la que se delega la gestión de la política del estado y del gobierno en cuanto a la atención estomatológica en el país.

En la década del 70', se consolidó el Sistema Nacional de Salud (SNS), único, eliminando la dispersión de funciones e instituciones sanitarias. La dirección de Estomatología inició un proceso de descentralización administrativa y ejecutiva a nivel provincial y municipal, respondiendo a la división político-administrativa del país. De esta forma sumada la desconcentración de recursos, se incrementó la capacidad de respuesta a los problemas de salud bucal con vinculación a los policlínicos que pasaron a ser comunitarios, facilitando la participación de la población, sus organizaciones y los sectores de la sociedad. A finales de esta década se instaura el modelo de Estomatología en la Comunidad, al que se incorporan nuevos métodos de trabajo, la docencia y la investigación en el primer nivel de atención.

En la década de los $80^{\prime}$, se establece el modelo de Estomatología General Integral (EGI), que se articula con el modelo de medicina familiar y el programa del Médico y Enfermera de la familia (1984), la atención de la población se organiza por consultorios de la familia, lo que incrementó la universalidad, la igualdad y la cobertura. En la década de los 90', se efectuaron reajustes encaminados a asegurar la atención en condiciones socio-económicas especiales. 
Las mejoras de la organización económico-social en la década $2000^{\prime}$, posibilitaron el remozamiento y construcción de servicios estomatológicos, asimismo mantener la estabilidad de los productos dentales. A partir de la década del $2010^{\prime}$, se introducen transformaciones encaminadas al aumento de la eficiencia, eficacia y efectividad, la reivindicación de los métodos clínico y epidemiológico, la mayor satisfacción de la población con los servicios y el incremento del control, también se trabaja en la preparación de directivos.

La red de instituciones que conforman el subsistema de atención estomatológica (clínicas estomatológicas, departamentos en policlínicos, hospitales, servicios en centros escolares, laborales entre otros), funciona acorde a lo reglamentado en el Plan Director de cada institución, el Reglamento General de Servicios Estomatológicos, el Manual de Organización y Procedimientos, las Guías Prácticas Clínicas y el Programa de Calidad ${ }^{13}$, formando parte de la red de servicios de salud integrados, integrales, generales y especializados con proyección comunitaria y participación social.

La estrategia de salud bucal en Cuba se establece por los principios rectores del SNS los cuáles son: universalidad, pues abarca a toda la población; regionalización, porque la red de servicios y las acciones se adecuan a la oferta asistencial a cada territorio; carácter estatal, ya que se subordina al estado y a sus órganos de gobierno; acceso gratuito a todos los servicios, orientación profiláctica por la prioridad al componente promocional y preventivo; la aplicación de los adelantos de la ciencia y la técnica socialmente adecuados y económicamente viables; la participación de la comunidad (familias, grupos sociales e individuos) contribuyendo a las acciones; la intersectorialidad, para afrontar los problemas en los que están implicados otros sectores socio-económicos; y la colaboración internacional, fuera del contexto nacional desde 1963.

Estos principios muestran el carácter integrador, inclusivo y solidario de la estrategia en respuesta a las legislaciones vigentes ${ }^{14,15}$.

\section{Programas y acciones para la salud bucal en Cuba}

Los programas para la atención estomatológica han facilitado la conducción de la estrategia de Estomatología a escala nacional, el primer programa básico de atención estomatológica (década de los años 60'), persiguió enfrentar el daño acumulado y a las extracciones dentarias como principal solución, estas acciones formaron parte de otras contra las enfermedades infectocontagiosas frecuentes (desnutrición por defecto, parasitismo, anemia etc.), y los estomatólogos integraron el equipo de salud conjuntamente con médicos generales, auxiliares de enfermería, trabajadores sanitarios, sociales y estadísticos 16. En 1969 comenzó la aplicación de laca flúor cada 6 meses a los niños de 2 a 5 años de edad, tanto los que estaban en su hogar como los asistentes a círculos infantiles. Luego en la década del 70', se adicionan los enjuagatorios de flúor al $0,2 \%$ cada 15 días a los escolares hasta los 11 años.
En los años 80, se introducen programas específicos de atención estomatológica a la población menor de 15 años, a la población con retraso mental, de diagnóstico precoz del cáncer bucal y de atención al adulto que operaban dentro del modelo de Estomatología General Integral ${ }^{13}$.

Respondiendo al modelo de atención de medicina familiar los programas orientados a grupos de población específicos se integraron en el "Programa Nacional de Atención Estomatológica Integral" en el año 1992, dicho programa fue revisado en 2002 y 2009 , en respuesta a la reorganización, las proyecciones y la incorporación de nuevos profesionales egresados de la Licenciatura en Tecnología de la Salud en los perfiles de atención estomatológica y prótesis dental.

Con el propósito de perfeccionar la atención de salud bucal en todo el país el programa agrupa acciones de salud integrales por objetivos que se resumen a continuación ${ }^{13}$.

- Ofrecer atención estomatológica primaria en la comunidad, mediante la vinculación entre el equipo de Estomatología (estomatólogos, licenciados en Atención Estomatológica y técnicos en Atención Estomatológica), con el Equipo Básico de Salud (médico y enfermera de la familia) y el Grupo Básico de Trabajo (personal de diferentes especialidades médicas, representantes del gobierno local y otros sectores políticos y de masas). Se orienta concertar el Análisis de la Situación de Salud (ASIS), de cada área.

- Incrementar la población sana, mediante acciones de promoción de salud (consejerías de salud) y educación para la salud individuales y colectivas.

- Disminuir la morbilidad de enfermedades bucodentales, mediante acciones de control de los factores de riesgo y detección temprana de problemas.

- Realizar acciones de curación (conservadoras), garantizando la cobertura al $100 \%$ de la población con necesidades y la continuidad de la atención hasta su total curación a través de equipos multidisciplinarios e interconsultas de ser necesario.

- Realizar acciones de rehabilitación estomatológica dando cobertura al mayor por ciento de población que lo requiera, priorizando en caso de limitaciones de recursos.

- Garantizar la calidad de la atención estomatológica integral y el cumplimiento de los principios bioéticos, mediante el análisis periódico de la calidad y la toma de medidas oportunas por la dirección.

- Disminuir la morbimortalidad por cáncer bucal mediante el pesquisaje activo anual a toda la población de 15 y más años de edad, el examen a la totalidad de la población que acuda a los servicios estomatológicos, la eliminación de hábitos lesivos a la mucosa bucal. En caso de sospecha remisión al servicio de 
cirugía maxilofacial correspondiente (según modelo establecido), dispensarizar, ofrecer atención estomatológica integral priorizada y dar seguimiento hasta la recuperación o fallecimiento, caso que lleva discusión.

- Brindar atención estomatológica integral a la población con discapacidad física y mental, dispensarizar de conjunto con el médico de familia y decidir el método de curación y rehabilitación convencional u hospitalaria.

- Brindar servicios de urgencia en Estomatología mediante guardia física del personal, que garantice la atención ininterrumpida.

- Desarrollar la Estrategia de Estomatología mediante investigaciones que contribuyan a resolver problemas.

- Promover el perfeccionamiento profesional y la actualización de todo el equipo de Estomatología así como el intercambio con expertos nacionales e internacionales.

Entre las principales líneas de acción para lograr los objetivos de salud bucal propuestas se hallan, las consejerías de salud bucal en las que la población recibe información y se establece comunicación acerca de la importancia de preservarla mediante la adopción de comportamientos saludables; el control de placa dentobacteriana a toda la población que asiste a los servicios, a los niños de 2 a 4 años y a los escolares en sus instituciones hasta alcanzar mejoría del nivel de higiene bucal; la realización de enjuagatorios bucales de fluoruro de sodio al $0,2 \%$ quincenales en las edades de 5 a 18 años, las aplicaciones de laca flúor semestrales a los niños de 2 a 4 años, 6 y 10 años, a los discapacitados y a los niños con elevado número de caries; la promoción del cepillado en los centros escolares; la detección temprana de hábitos inadecuados; el pesquisaje activo del cáncer bucal, la promoción del autoexamen bucal; el examen clínico antes de 3 meses de edad y la atención estomatológica priorizada a grupos poblacionales más vulnerables como las embarazadas, madres con niños menores de un año, población menor de 19 años, discapacitados físicos y mentales, población adulta mayor - 60 y más años de edad -, personas con lesiones estomatológicas potencialmente malignas y personas con enfermedades crónicas.

La vigilancia de salud bucal posibilita identificar las variaciones en la morbilidad bucodental y los factores que la condicionan, asimismo detectar alarmas. Esta vigilancia se efectúa a través de, estudios epidemiológicos nacionales para el seguimiento al estado de salud bucodental desde el año 1984; el sistema de vigilancia activa en sitios centinelas para el monitoreo de eventos de morbilidad críticos que pudieran paralizar los servicios desde el año 1999; y el sistema de información estadística continua desde los servicios hasta el nivel central para la evaluación del sistema de gestión ${ }^{13}$. A ello se le añaden resultados de investigaciones realizadas, incluidas las encargadas por el nivel rector.

\section{Formación de recursos humanos}

El Ministerio de Salud Pública se ocupa de la formación y perfeccionamiento de todos sus recursos humanos siguiendo orientaciones metodológicas del Ministerio de Educación Superior. Los recursos humanos de la Estomatología se cursan en 4 Facultades de Estomatología (la Habana, Villa Clara, Camagüey y Santiago de Cuba), asimismo en Facultades y Universidades de Ciencias Médicas de todo el país ${ }^{17}$. La carrera de Estomatología ha sido sometida a evaluación externa con el Sistema de Evaluación y Acreditación de Carreras Universitarias (SEA-CU), en la actualidad hay tres de excelencia y siete certificadas ${ }^{18}$.

Los cambios en los modelos de atención han conducido al perfeccionamiento del curriculum y planes de estudio. La formación profesional integra docencia, asistencia, investigación y la educación en el trabajo. Por eso el perfil profesional es amplio y se prepara para el desempeño tanto en lo científico-técnico como en la ética, humanismo, solidaridad y otros valores que favorecen el actuar por el derecho a la salud de todos.

En la formación del estomatólogo general básico la Estomatología General Integral es la disciplina integradora, apoyada en el análisis de la situación de salud, lo cual propicia que los estudiantes se eduquen y actúen teniendo en cuenta los determinantes sociales de la salud ${ }^{19-21}$, aportando así a la salud de la población en escenarios reales. Concluidos estos estudios se cursa en dos años la Especialidad Estomatología General Integral (EGI), - creada en 1998 y generalizada en el 2004-, que corresponde al primer nivel de atención responsabilizado con la Atención Estomatológica Integral a la población dentro del marco de las funciones de salud pública-, de manera que su práctica profesional promueva la identificación, control y vigilancia de los factores asociados a la situación de salud bucal y consecuentemente contribuya a la elevación de la cultura de salud, reportando incremento del nivel de salud de la población de los consultorios de la familia asignados.

A diferencia de la Estomatología General No Integral que se limita al diagnóstico clínico y el tratamiento de enfermos, habitualmente fragmentado por excesivas especialidades. La EGI debe actuar según planificación estratégica basado en las necesidades de la población, capital humano y recursos de cada contexto, y enfocarse a producir salud poblacional con todos los implicados. En consecuencia, la práctica profesional del EGI requiere integración intradisciplinaria con las especialidades estomatológicas (Ortodoncia, Prótesis, Periodoncia y Cirugía Maxilofacial), e integración interdisciplinaria con el Médico General Integral y Enfermera de la Familia, así como con profesionales y técnicos de otras ramas de la medicina y psicólogos, también necesita coordinación con otros sectores de la sociedad implicados en los problemas de salud bucal de cada comunidad de manera que se establezca un sistema continuo de atención dentro de la atención primaria de salud, que se concibe como estrategia. 
Los doctorados, maestrías, y otras modalidades de estudios de postgrado fortalecen el capital humano que constituye el principal recurso.

Desde 1959 al 2018 se han graduado 28207 estomatólogos. En la actualidad se cuenta con un capital humano de 18910 estomatólogos, distribuidos por todas las provincias; 16,8 estomatólogos por 10000 habitantes y 595 habitantes por estomatólogo ${ }^{22}$.

\section{Resultados de la estrategia estomatología}

En respuesta al planteamiento de "Salud para todos", se alcanzaron las metas de salud bucal 1 en el año 1998 con $55 \%$ de $50 \%$ establecido, la meta 2 en el año 1989 con 2,93 del promedio establecido de 3, la meta 3 en el 2005 con $89,9 \%$ de $85 \%$ establecido, la meta 4 con 5,1 del promedio establecido de 6,3 y la meta 5 en 1998 con 20,6 del promedio establecido de $24^{13,18}$.

En cuanto a la actividad asistencial, el número de consultas y su promedio por habitante ha ido en incremento sostenido desde el año 2012, correspondiendo la mayor parte a consultas de Estomatología General Integral donde también se ofrecen tratamientos de Medicina Natural y Tradicional. Respecto a la rehabilitación de la cara y prótesis bucomaxilofacial en 2017 y 2018 se concluyeron más del 90,0\% de los tratamientos. Se mejoró la disponibilidad técnica de equipos para los servicios de Cirugía Maxilofacial en los hospitales de todas las provincias ${ }^{22}$.

Los resultados se deben más que todo al reconocimiento del derecho universal a la salud de la población, ${ }^{23}$ aunado a las políticas de justicia y equidad que han reportado al desarrollo humano y sostenible ${ }^{24,25}$.

\section{Conclusiones}

En Cuba la salud bucal se aborda como bien social y derecho humano, dentro de la política de salud pública orientada a la salud, bienestar y la calidad de vida, a través de la participación mancomunada de la sociedad y el estado en consonancia con la determinación social de cada momento y con las recomendaciones de las organizaciones rectoras internacionales. No obstante los avances, están presentes retos para el perfeccionamiento en el ámbito de la metodología de trabajo del EGI, la calidad de los servicios, la formación de doctores en ciencias, la producción científica, las capacidades para la promoción de salud y la prevención de enfermedades bucales. Asimismo para mantener el suministro de materiales, equipos, tecnologías biomédicas y servicios adecuados en un contexto muy adverso, por el reforzamiento del bloqueo económico y comercial de los Estados Unidos hacia Cuba y su aplicación extraterritorial, que limita la adquisición de los recursos materiales y equipos, que requiere un sistema de salud de cobertura y acceso universal con financiamiento estatal.

\section{Referencias bibliográficas}

1. OMS. Nota Descriptiva No. 323. [Internet]. 2015 [citado 6 de marzo de 2020]. Disponible en: http://www. who.int/mediacentre/factsheets/fs323/es/.
2. Castell-Florit Serrate P, Gispert Abreu E, Lozano Lefrán A. Cobertura universal de salud y conciliación de criterios en base a la experiencia cubana. Rev Cubana Salud Pública [Internet]. 2016 [citado 6 de marzo de 2020]; 41(1). Disponible en: http://www.revsaludpublica.sld. cu/index.php/spu/article/view/634.

3. Rueda Martínez G, Albuquerque A. La salud bucal como derecho humano y bien ético. Rev Latinoam Bioet [Internet]. 2017 [citado 17 de febrero de 2020]; 25(2):224-233. Disponible en: http://www.scielo.br/scielo.php?script=sci_arttext\&pid=S1983-80422017000200224\&lng=en . https://doi.org/10.1590/1983-80422017252182.

4. Petersen PE. Improvement of global oral health - the leadership role of the World Health Organization. Community Dental Health [Internet]. 2010 [citado 6 de marzo de 2020]; 27: 194-199). Disponible en: https://pdfs.semanticscholar.org/850c/3a98b568cd91556886465f34e7d410b2c6dd.pdf.

5. Petersen P. E. Strengthening of Oral Health Systems: Oral Health through Primary Health Care. Medical Principles and Practice [Internet]. 2014 [citado 6 de marzo de 2020]; 23(1): 3-9 Disponible en: https://doi. org/10.1159/000356937.

6. Rueda Martínez G, Albuquerque A. El derecho a la salud bucal en la Declaración de Liverpool. Rev. Bioét [Internet]. 2017 [citado 17 de febrero de 2020]; 25(2): 224-233. Disponible en: http://www.scielo.br/scielo.php?script=sci_arttext\&pid=S1983-80422017000200224\&lng=en. https://doi.org/10.1590/1983-80422017252182.

7. Gispert Abreu E de los Á, Bécquer Águila J L. Salud bucal poblacional, una prioridad en todas las políticas. Rev Cubana Estomatol [Internet]. 2015 [citado 6 de marzo de 2020]; 52(3): 231-234. Disponible en: http://scielo.sld.cu/scielo.php?script=sci_arttext\&pi$\mathrm{d}=$ S0034-75072015000300001\&lng=es.

8. FDI. Visión 2020. Delinear el futuro de la salud bucodental. [Internet]. 2012 [citado 17 de febrero de 2020]. Disponible en: www.fdiwordental.org/library.

9. Cohen L, Johnson NW, Fejerskov O, Dahlen G, Manji F, Escobar-Rojas A. La Odontología en crisis: Tiempo para cambiar. La declaración de La Cascada: nos preocupa que la odontología mundial haya perdido su camino. Rev CES Odont [Internet]. 2018 [citado 6 de marzo de 2020]; 31(2): 1-5. Disponible en: http://www.scielo.org. co/pdf/ceso/v31n2/0120-971X-ceso-31-02-1.pdf.

10. FDI. El Desafío de las Enfermedades Bucodentales Una llamada a la acción global. Atlas de Salud Bucodental [Internet]. 2015 [citado 6 de marzo de 2020]. Disponible en: http://www.fdiworldental.org/media/84768/ book_spreads_oh2_spanish.pdf.

11. Suárez-Ponce D, Watanabe-Velásquez R, Zambrano-De la Peńa S, Anglas-Machacuay A, Romero-Álvarez V, Montano-Rubín De Celis Y. Bioética, principios y dilemas éticos en Odontología. Odontol Sanmarquina [Internet]. 2016 [citado 17 de febrero de 2020]; 19 (2):3340. Disponible en:revistasinvestigacion.unmsm.edu.pe/ index.php/odont/article/download/12919/11538.

12. OPS. Propuesta de plan regional decenal sobre salud bucodental para las Américas: Informe final. 160. a Sesión del Comité Ejecutivo. CE160/INF/7 [Internet]. 2017 [citado 6 de marzo de 2020]. Disponible en: https:// 
iris.paho.org/bitstream/handle/10665.2/34217/CE160INF-7-s.pdf;jsessionid=DD351318B80150D79EE820AABDCAD440? sequence $=2$.

13. MINSAP. Dirección Nacional de Estomatología. Programa Nacional de Atención Estomatológica Integral a la población. Editorial DAMPSA. La Habana; 2013.

14. Ley No 41/1983 [Internet].1983 [citado 28 de Abril de 2019]. Disponible en: http://legislacion.sld.cu/index. php?P=FullRecord \&ID=2.

15. Constitución de la República de Cuba. [Internet]. 2019 [citado 28 de abril de 2019]. Disponible en: http://media.cubadebate.cu/wp-content/uploads/2019/01/Constitucion-Cuba-2019.pdf.

16. González Cárdenas LT, Cuesta Mejías L, Pérez Perea L, Presno Labrador MC, Fernández Díaz IE, Pérez Díaz TC, Guerrero Chacón SE, Pérez Charbonier C. El Programa del médico y enfermera de la familia: desarrollo del modelo de atención médica en Cuba. Rev Panam Salud Pública [Internet]. 2018 [citado 17 febrero de 2020]; 42:e31. Disponible en: https://doi.org/10.26633/ RPSP.2018.31

17. Vela-Valdés J, Salas-Perea RS, Quintana-Galende ML, Pujals-Victoria N, González Pérez J, Díaz Hernández L, et al. Formación del capital humano para la salud en Cuba. Rev Panam Salud Pública [Internet]. 2018 [citado 17 febrero de 2020]; 42:e33 Disponible en: https://doi. org/10.26633/RPSP.2018.33

18. González Longoria M de la CB, Grau León IB, Urbizo Vélez J, Soto Cantero LA, Sosa Rosales M. Formación de recursos humanos en estomatología, su impacto en la salud bucal de la población. Educ Med Super [Internet]. 2017 [citado 17 febrero de 2020]; 31(2). Disponible en: http://scielo.sld.cu/scielo.php?script=sci_arttext\&pi$\mathrm{d}=$ S0864-21412017000200020\&lng=es.

19. Rosales Reyes SA. Las determinantes sociales en el plan d de la carrera de estomatología. Cuba Conference Paper [Internet]. 2015 [citado 17 febrero de 2020]. Disponible en: https:/www.researchgate.net/publication/319068645
20. González Rodríguez S, Pedroso Ramos L, Rosales Reyes SA. Contribución de la Disciplina Estomatología Integral al Análisis de la Situación de Salud Bucal Revista Habanera de Ciencias Médicas [Internet]. 2015 [citado 17 febrero de 2020]; 14(2): 227-236. Disponible en: http://www.redalyc.org/articulo.oa?id=180438823012

21. Rodríguez Fuentes S, Breijo Worosz T, Gato Armas CA. Estrategia para lograr la profesionalización en función del desarrollo social comunitario. Avances [Internet]. 2019 [citado 17 febrero de 2020]; 21(2):208-219. Disponible en: http://www.ciget.pinar.cu/ojs/index.php/ publicaciones/article/view/436

22. Ministerio de Salud Pública (MINSAP). Dirección de Registros Médicos y Estadísticas de Salud. Anuario Estadístico de Salud [Internet]. 2019 [citado 17 febrero de 2020].disponible en: http://files.sld.cu/bvscuba/files/2019/04/Anuario-Electr\%C3\%B3nico-Espa\%C3\%B1ol-2018-ed-2019.pdf

23. Botero JC, Puerta E, Lumertz J, Sonaglio R, Melo R, Rocha C. Perspectivas teórico-prácticas sobre promoción de la salud en Colombia, Cuba y Costa Rica: revisión integrativa. [Internet].2016 [citado $17 \mathrm{fe}$ brero de 2020]; 21(2): 59-73.Disponible en: http:// www.scielo.org.co/scielo.php?script=sci_arttext\&pi$\mathrm{d}=$ S0121-75772016000200005\&lng=en.

24. PNUD. Informe sobre Desarrollo Humano [Internet]. 2019 [citado 10 marzo de 2020]. Disponible en: http:// www.www.undp.org.

25. Hickel J. The Sustainable Development Index: Measuring the Ecological Efficiency of Human Development in the Anthropocene, Ecological Economics [Internet]. 2020 [citado 10 marzo de 2020]. Disponible en: http:// www.sustainabledevelopmentindex.org. 